\title{
Rapid, Simple, and Sensitive Spectrofluorimetric Method for the Estimation of Ganciclovir in Bulk and Pharmaceutical Formulations
}

\author{
Garima Balwani, Emil Joseph, Satish Reddi, Vibhu Nagpal, and Ranendra N. Saha \\ Department of Pharmacy, BITS Pilani, Pilani Campus, Rajasthan 333031, India
}

Correspondence should be addressed to Garima Balwani; garimabalwani@gmail.com

Received 17 May 2013; Accepted 18 September 2013

Academic Editor: Austin Nevin

Copyright (C) 2013 Garima Balwani et al. This is an open access article distributed under the Creative Commons Attribution License, which permits unrestricted use, distribution, and reproduction in any medium, provided the original work is properly cited.

\begin{abstract}
A new, simple, rapid, sensitive, accurate, and affordable spectrofluorimetric method was developed and validated for the estimation of ganciclovir in bulk as well as in marketed formulations. The method was based on measuring the native fluorescence of ganciclovir in $0.2 \mathrm{M}$ hydrochloric acid buffer of $\mathrm{pH} 1.2$ at $374 \mathrm{~nm}$ after excitation at $257 \mathrm{~nm}$. The calibration graph was found to be rectilinear in the concentration range of $0.25-2.00 \mu \mathrm{g} \mathrm{mL}^{-1}$. The limit of quantification and limit of detection were found to be $0.029 \mu \mathrm{g} \mathrm{mL}$ and $0.010 \mu \mathrm{g} \mathrm{mL}^{-1}$, respectively. The method was fully validated for various parameters according to ICH guidelines. The results demonstrated that the procedure is accurate, precise, and reproducible (relative standard deviation $<2 \%$ ) and can be successfully applied for the determination of ganciclovir in its commercial capsules with average percentage recovery of 101.31 \pm 0.90 .
\end{abstract}

\section{Introduction}

Ganciclovir, 9-[(1,3-dihydroxy-2-propoxy) methyl]guanine, is a well-known anti-viral agent. It is an acyclic nucleoside analogue of 2-deoxy guanosine that inhibits replication of herpes virus, with substantially increased antiviral activity against cytomegalovirus (CMV) $[1,2]$. CMV infections are the main cause of morbidity and mortality in immunocompromised patients, mainly in those with the acquired immunodeficiency syndrome (AIDS), congenital immunodeficiency, or in individuals following organ transplantation [3]. In these patients ganciclovir is one of the preferred drugs for treatment.

An extensive review of the literature has not revealed any simple spectrofluorimetric method for the estimation of ganciclovir in the pure form and in pharmaceutical dosage forms. Several high performance liquid chromatography (HPLC) and few radioimmunoassay and enzyme-linked immunosorbent assays have been reported for the quantification of this drug [4]. Most of these methods are developed to quantify ganciclovir in biological fluids like plasma, serum, and tissues [5-12]. There are also few reports dealing with the application of HPLC in the estimation of ganciclovir in bulk and pharmaceuticals $[13,14]$, but these methods are moderately sensitive and have significant disadvantages due to the high cost, large number of steps, and lengthy time required to obtain final quantitative results.

In this study, a spectrofluorimetric method was developed for estimation of ganciclovir, as it can estimate analytes at very low concentrations of nanogram level and is fast and economic for routine analysis. In the developed method, no extraction step was utilized, thus reducing the time and error involved in the estimation. The proposed method was fully validated according to ICH guidelines, and successfully applied for the determination of the drug in its capsules.

\section{Experimental}

2.1. Apparatus. A Shimadzu (Japan) Spectrofluorophotometer Model RF-5301PC equipped with xenon lamp and $3 \mathrm{~mm}$ quartz cells was used for the estimation process. $\mathrm{pH}$ measurements were made with Eutech $\mathrm{pH}$ Meter (India).

2.2. Materials and Reagents. Ganciclovir was provided by Ranbaxy Laboratories Limited, Gurgaon, India, as a generous gift sample. Commercial product Ganguard capsules (labeled 
to contain $250 \mathrm{mg}$ ganciclovir per capsule) were obtained from a local drug store. All other chemicals and reagents used were of analytical grade.

2.3. Stock and Standard Solution. A stock solution of $100.0 \mu \mathrm{g} \mathrm{mL}^{-1}$ of ganciclovir was prepared in water. A standard solution of $5.0 \mu \mathrm{g} \mathrm{mL}^{-1}$ of the drug was prepared further by dilution with $0.2 \mathrm{M}$ hydrochloric acid buffer $(\mathrm{pH} 1.2)$ medium. The stock solution was found to be stable for at least 1 week when kept in the refrigerator.

2.4. Construction of Calibration Curve. Aliquots of ganciclovir working standard solution were transferred into a series of $2 \mathrm{~mL}$ centrifuge tubes to make nine different sets of final concentrations of $0.25-2.00 \mu \mathrm{g} \mathrm{mL}^{-1}$ and were diluted to the mark with hydrochloric acid buffer of $\mathrm{pH} 1.2$ and mixed well. The fluorescence intensities were measured at $374 \mathrm{~nm}$ after excitation at $257 \mathrm{~nm}$. The relative fluorescence intensities (RFI) were plotted against the final concentration of the drug $\left(\mu \mathrm{g} \mathrm{mL}^{-1}\right)$ to get the calibration curve, and the corresponding regression equation was derived.

\subsection{Validation of the Method}

2.5.1. Linearity. To establish linearity of the proposed method, nine separate series of solutions of the drug (0.25$2 \mu \mathrm{g} \mathrm{mL}^{-1}$ in the hydrochloric acid buffer of $\mathrm{pH} 1.2$ ) were prepared from the stock solution and analyzed. Least square regression analysis was done for the obtained data. ANOVA test (one-way) was performed based on the fluorescence intensity values observed for each drug concentration during the replicate measurement of the standard solutions.

2.5.2. Limit of Quantification and Limit of Detection. The limit of quantification (LOQ) and limit of detection (LOD) were determined according to ICH Q2 (R1) recommendations [15]. LOQ and LOD were calculated as $10 \times S_{a} / b$ and $3.3 \times S_{a} / b$, respectively, where $S_{a}$ is the standard deviation of $y$-intercept of regression equation and $b$ is the slope of the calibration curve.

2.5.3. Accuracy and Precision. As a part of determining, the accuracy of the proposed method, different levels of drug concentrations, namely, low quality control (LQC), medium quality control (MQC), and high quality control (HQC), were prepared from independent stock solution and analyzed $(N=$ 9). Accuracy was assessed as the percentage relative error and mean percentage recovery for each of the quality control samples.

Repeatability was determined by using different levels of drug concentrations prepared from independent stock solution and was analysed $(N=9)$. Interday and intraday variation and instrument variation were taken to determine intermediate precision of the proposed method. Different levels of drug concentrations in triplicate were prepared, three different times in a day, and studied for intraday variation. Same protocol was followed for three different days

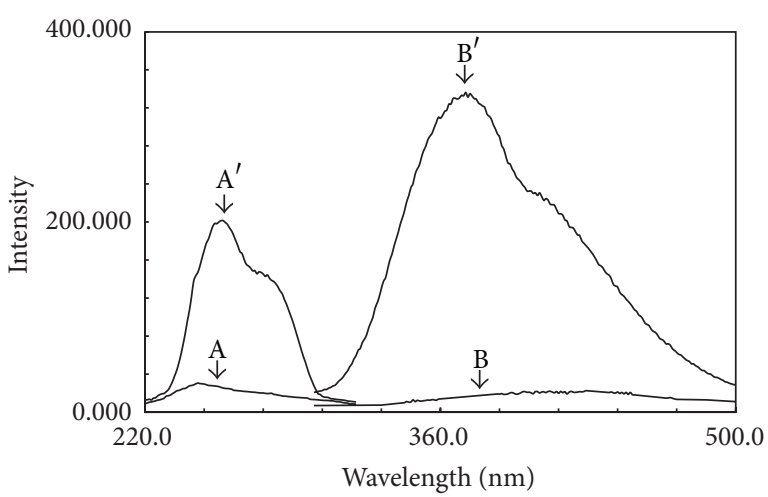

FIGURE 1: Fluorescence spectra of (A, B) hydrochloric acid buffer of $\mathrm{pH} 1.2$ and $\left(\mathrm{A}^{\prime}, \mathrm{B}^{\prime}\right)$ ganciclovir $\left(1.1 \mu \mathrm{g} \mathrm{mL} \mathrm{m}^{-1}\right)$ in hydrochloric acid buffer of $\mathrm{pH}$ 1.2. Where: $\left(\mathrm{A}, \mathrm{A}^{\prime}\right)$ are the excitation spectra and $\left(\mathrm{B}, \mathrm{B}^{\prime}\right)$ are the emission spectra.

to study interday variation $(N=27)$. The relative standard deviation (RSD) (in \%) of the predicted concentrations from the regression equation was taken as the measure of precision.

2.5.4. Specificity. Drug concentration of $1 \mu \mathrm{g} \mathrm{mL}^{-1}$ was prepared independently from pure drug stock and commercial sample stock in selected medium and was analysed $(N=$ 5). Paired $t$-test and variance ratio $F$ test at $95 \%$ level of significance were performed to compare the means of percent recovery values.

2.5.5. Robustness. Robustness was examined by evaluating the influence of small variation in the experimental parameters on the analytical performance of the method. It was determined by (a) changing $\mathrm{pH}$ of the medium by \pm 0.2 units and (b) stability of ganciclovir in the selected medium at room temperature for 48 hours. Three different concentrations (LQC, MQC, and HQC) were prepared in the media with different $\mathrm{pH}$, and mean percentage recovery was determined for each sample.

2.6. Assay Procedure for Capsules. Ten capsules were weighed, and their contents were taken and thoroughly mixed. Amount of the powder equivalent to $50 \mathrm{mg}$ of ganciclovir was taken, mixed with the selected medium to prepare a $100 \mu \mathrm{g} \mathrm{mL}^{-1}$ concentration, sonicated for 30 minutes, and filtered through Whatman filter paper number 40. The filtrate was suitably diluted to prepare a $1 \mu \mathrm{g} \mathrm{mL}^{-1}$ concentration in the medium. The procedure described under "construction of calibration curve" was followed. The nominal content of the capsules was calculated using the regression equation.

\section{Results and Discussion}

Ganciclovir was found to exhibit intense native fluorescence in $0.2 \mathrm{M}$ hydrochloric acid buffer $(\mathrm{pH} 1.2)$ at $374 \mathrm{~nm}$ after excitation at $257 \mathrm{~nm}$ (Figure 1). 


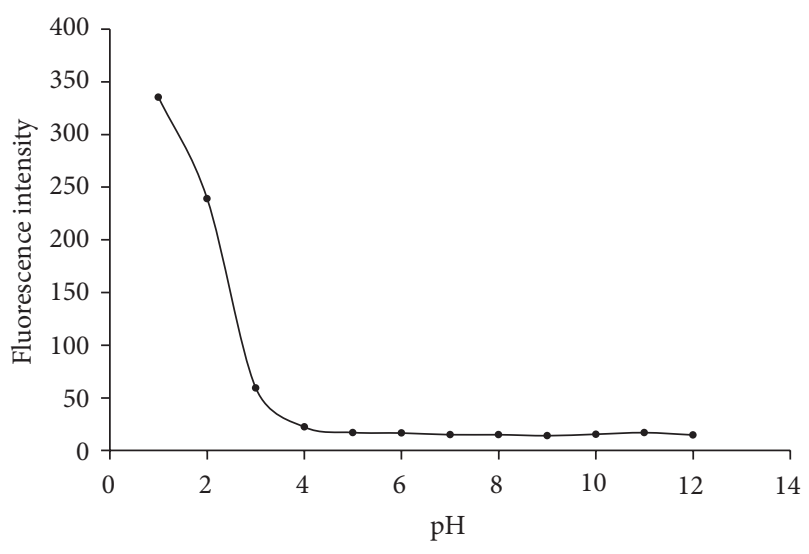

Figure 2: Effect of $\mathrm{pH}$ on fluorescence intensity.

TABle 1: Performance data for the proposed spectrofluorimetric method for determination of ganciclovir.

\begin{tabular}{lc}
\hline Parameter & Results \\
\hline Concentration range $\left(\mu \mathrm{g} \mathrm{mL}^{-1}\right)$ & $0.25-2.00$ \\
Regression coefficient $\left(r^{2}\right)$ & 1.00 \\
Slope & 267 \\
Intercept & 3.5 \\
$S_{y / x}$ & 0.9866 \\
$S_{a}$ & 0.7688 \\
$S_{b}$ & 0.6089 \\
Calculated $F$ value & 0.001 \\
$(\text { Critical } F \text { value })^{\mathrm{a}}$ & $(2.032)$ \\
\hline
\end{tabular}

$S_{y / x}$ : standard deviation of the residuals. $S_{a}$ : standard deviation of the intercept. $S_{b}$ : standard deviation of the slope.

${ }^{\text {a }}$ Theoretical value of $F(8,63)$ based on one-way ANOVA test at $P=0.05$ level of significance.

3.1. Effect of $\mathrm{pH}$ on Fluorescence. The influence of $\mathrm{pH}$ on the native fluorescence intensity of ganciclovir was studied. The results showed that the native fluorescence intensity of ganciclovir decreased with increasing the $\mathrm{pH}$ of the buffer up to 12.0 (Figure 2). Therefore, $0.2 \mathrm{M}$ hydrochloric acid buffer $\mathrm{pH} 1.2$ could be used for ganciclovir determination as it showed maximum and stable fluorescence intensity at this $\mathrm{pH}$.

3.2. Validation of the Method. The validity of the method was checked by testing linearity, sensitivity, limit of detection, limit of quantitation, specificity, accuracy, precision, and robustness according to $\mathrm{ICH}$ recommendations

3.2.1. Linearity. Using the above procedures it was found that there is a linear relationship between RFI and concentrations over the range of $0.25-2.00 \mu \mathrm{g} \mathrm{m}^{-1}$. Linear regression analysis of the data gave the equation RFI $=3.5+267 \times C$, where $C$ is the final concentration of ganciclovir in $\mu \mathrm{g} \mathrm{m}^{-1}$. Statistical evaluation of the regression data for ganciclovir was performed, and the resultant standard deviation of the residual $\left(S_{y / x}\right)$, standard deviation of the intercept $\left(S_{a}\right)$, and
TABLE 2: Evaluation of accuracy of the proposed method for ganciclovir determination in pure form.

\begin{tabular}{lccc}
\hline $\begin{array}{l}\text { Conc. taken } \\
\left(\mu \mathrm{gL}^{-1}\right)\end{array}$ & $\begin{array}{c}\text { Conc. found } \\
\left(\mu \mathrm{gL}^{-1}\right) \pm \\
\text { S.D. }\end{array}$ & \% Recovery \pm S.D. & Accuracy ${ }^{*} \pm$ S.D. \\
\hline 0.4 & $0.406 \pm 0.01$ & $101.61 \pm 2.41$ & $1.61 \pm 2.41$ \\
1.1 & $1.117 \pm 0.03$ & $101.52 \pm 2.78$ & $1.52 \pm 2.78$ \\
1.8 & $1.817 \pm 0.02$ & $100.96 \pm 1.31$ & $0.96 \pm 1.31$ \\
\hline
\end{tabular}

Each result is the average of six separate determinations.

${ }^{*}$ Accuracy is given in \% relative error $=100 \times[$ (predicted concentration nominal concentration)/nominal concentration].

TABLE 3: Results of standard addition method.

\begin{tabular}{lccc}
\hline $\begin{array}{l}\text { Amount in } \\
\text { formulation } \\
(\mu \mathrm{g})\end{array}$ & $\begin{array}{c}\text { Amount added } \\
(\mu \mathrm{g})\end{array}$ & $\begin{array}{c}\text { Amount } \\
\text { found } \pm \text { S.D. } \\
(\mu \mathrm{g})\end{array}$ & $\begin{array}{c}\text { \% Recovery } \pm \\
\text { S.D. }\end{array}$ \\
\hline 7.56 & 2.0 & $9.52 \pm 0.11$ & $99.54 \pm 1.19$ \\
7.56 & 2.5 & $10.08 \pm 0.12$ & $100.24 \pm 1.22$ \\
7.56 & 3.0 & $10.55 \pm 0.09$ & $99.93 \pm 0.86$ \\
\hline
\end{tabular}

Each result is the average of three separate determinations.

standard deviation of the slope $\left(S_{b}\right)$ are given in Table 1 . The very low values of these parameters point out low scattering of the points around the calibration curve, thus, indicating the high accuracy and high precision of the method. Goodness of fit of regression equation was supported by high regression coefficient value of 1.0 and less calculated $F$ value.

3.2.2. $L O Q$ and $L O D$. The $L O Q$ was found to be $0.029 \mu \mathrm{g} \mathrm{mL}^{-1}$, and the LOD was found to be $0.010 \mu \mathrm{g} \mathrm{mL}^{-1}$, depicting the sensitivity of the proposed method.

3.2.3. Accuracy and Precision. Accuracy (given as $\%$ relative error) ranged from $1.00 \%$ to $1.64 \%$, (Table 2 ). The mean percentage recoveries of nearly $100 \%$ and low $\%$ RSD values for lower, intermediate, and higher concentrations revealed that any small change in the drug concentration in the solution can be accurately determined by this proposed method. The validity and reliability of the proposed method were evaluated by recovery studies of standard addition method (Table 3 ). In the intraday and interday precision study, the \% RSD values were found to be very small indicating reasonable repeatability of the proposed method (Table 4 ).

3.2.4. Specificity. The specificity of the proposed method was proven by the ability to determine ganciclovir in its capsules without interference from the common excipients. The emission spectrum of pure drug sample was matching the marketed formulation sample in the selected medium. The calculated $t$ value and variance ratio $F$ value were found to be less than those of the critical values, indicating that statistically there was no significant difference between mean fluorescence intensity of solutions prepared from pure drug sample and commercial formulation sample (Table 5). 
TABle 4: Precision data for the proposed method.

\begin{tabular}{lcc}
\hline Conc. $\left(\mu \mathrm{g} \mathrm{mL}^{-1}\right)$ & \% Recovery \pm S.D. & \% RSD \\
\hline \multicolumn{3}{c}{ Intraday precision } \\
0.4 & $100.39 \pm 1.40$ & 1.39 \\
1.1 & $100.99 \pm 1.57$ & 1.55 \\
1.8 & $100.15 \pm 1.55$ & 1.55 \\
\hline \multicolumn{3}{c}{ Interday precision } \\
0.4 & $100.73 \pm 1.51$ & 1.50 \\
1.1 & $100.85 \pm 1.38$ & 1.37 \\
1.8 & $100.55 \pm 1.02$ & 1.01 \\
\hline
\end{tabular}

Each result is the average of nine separate determinations.

TABLE 5: Determination of ganciclovir in commercial formulation and statistical comparison with the proposed method.

\begin{tabular}{lcc}
\hline $\begin{array}{l}\text { Conc. } \\
\left(\mu \mathrm{g} \mathrm{mL}^{-1}\right)\end{array}$ & \multicolumn{2}{c}{ \% Recovery \pm S.D. } \\
\hline 0.4 & $101.65 \pm 1.22$ & Pure drug sample \\
1.1 & $100.29 \pm 0.76$ & $100.74 \pm 0.59$ \\
1.8 & $101.98 \pm 0.04$ & $101.94 \pm 0.79$ \\
Mean \pm S.D. & $101.31 \pm 0.90$ & $100.17 \pm 2.11$ \\
$t$ & $0.84(2.78)^{*}$ & \\
$F$ & $0.18(19.0)^{*}$ & \\
\hline
\end{tabular}

Each result is the average of three separate determinations.

${ }^{*}$ Values between brackets are the tabulated $t$ and $F$ values, at $P=0.05$.

TABLE 6: Robustness data for the proposed method.

\begin{tabular}{lcc}
\hline Conc. $\left(\mu \mathrm{g} \mathrm{mL}^{-1}\right)$ & \% Recovery $( \pm$ S.D. $)$ & \% RSD \\
\hline \multicolumn{3}{c}{ pH 1.0} \\
0.4 & $97.92 \pm 0.68$ & 0.70 \\
1.1 & $98.19 \pm 1.37$ & 1.40 \\
1.8 & $99.70 \pm 0.61$ & 0.61 \\
\hline \multicolumn{3}{c}{$\mathrm{pH} 1.4$} \\
0.4 & $97.84 \pm 1.05$ & 1.07 \\
1.1 & $100.74 \pm 0.59$ & 0.59 \\
1.8 & $101.94 \pm 0.79$ & 0.78 \\
\hline \multicolumn{4}{c}{$101.74 \pm 1.08$} \\
0.4 & $101.99 \pm 0.89$ & 1.06 \\
1.1 & $100.74 \pm 0.14$ & 0.87 \\
1.8 & Stability after 48 hours at room temperature & 0.13 \\
\hline
\end{tabular}

Each result is the average of three separate determinations.

3.2.5. Robustness. It was found that none of the variables significantly affected the performance of the method as the recovery values were found to be from $97.84 \%$ to $101.94 \%$ (Table 6). Also the ganciclovir solution in the selected medium exhibited no changes in spectra and intensity for 48 hours when kept at room temperature. This provides an indication of the reliability and stability of the proposed method during the routine analysis.

3.3. Application of the Method. The proposed method was successfully applied to the analysis of ganciclovir in commercial capsules. The assay values of ganciclovir in the formulation ranged from $100.29 \%$ to $101.98 \%$ of the claimed amount with standard deviation of not more than 1.22 as shown in Table 5.

\section{Conclusions}

The proposed spectrofluorimetric method for the determination of ganciclovir was found to be sensitive, rapid, and simple. The method was validated and proven to be selective, accurate, precise, and robust, so it could be used for estimation of ganciclovir in bulk, pharmaceutical formulations, and different dissolution samples.

\section{Conflict of Interests}

The authors declare that there is no conflict of interests with any financial/commercial organization regarding the material discussed in the paper.

\section{Acknowledgments}

The authors are grateful to Ranbaxy Laboratories Limited (Gurgaon, India) for the gift sample of ganciclovir and the Department of Science and Technology (DST)-INSPIRE Program, New Delhi, India, for the financial support.

\section{References}

[1] M. A. Jacobson, "Treatment of cytomegalovirus retinitis in patients with the acquired immunodeficiency syndrome," The New England Journal of Medicine, vol. 337, no. 2, pp. 105-114, 1997.

[2] D. Faulds and R. C. Heel, "Ganciclovir. A review of its antiviral activity, pharmacokinetic properties and therapeutic efficacy in cytomegalovirus infection," Drugs, vol. 39, no. 4, pp. 597-638, 1990.

[3] V. C. Emery, "Investigation of CMV disease in immunocompromised patients," Journal of Clinical Pathology, vol. 54, no. 2, pp. 84-88, 2001.

[4] M. E. Bosch, A. J. R. Sánchez, F. S. Rojas, and C. B. Ojedac, "Ganciclovir: a review of its analytical determination," Asian Journal of Pharmaceutical Sciences, vol. 4, no. 4, pp. 254-264, 2009.

[5] A. Padullés, H. Colom, Y. Armendariz et al., "Determination of ganciclovir in human plasma by ultra performance liquid chromatography-UV detection," Clinical Biochemistry, vol. 45, no. 4-5, pp. 309-314, 2012.

[6] M. A. Campanero, B. Sadaba, E. García-Quetglas, and J. R. Azanza, "Development and validation of a sensitive method for the determination of ganciclovir in human plasma samples by reversed-phase high-performance liquid chromatography," Journal of Chromatography B, vol. 706, no. 2, pp. 311-317, 1998.

[7] D. Teshima, K. Otsubo, T. Yoshida, Y. Itoh, and R. Oishi, "A simple and simultaneous determination of acyclovir and ganciclovir in human plasma by high-performance liquid chromatography," Biomedical Chromatography, vol. 17, no. 8, pp. 500-503, 2003.

[8] F. Chu, C.-H. Kiang, M.-L. Sung, B. Huang, R. L. Reeve, and T. Tarnowski, "A rapid, sensitive HPLC method for the determination of ganciclovir in human plasma and serum," 
Journal of Pharmaceutical and Biomedical Analysis, vol. 21, no. 3, pp. 657-667, 1999.

[9] N. Perrottet, A. Beguin, P. Meylan et al., "Determination of aciclovir and ganciclovir in human plasma by liquid chromatography-spectrofluorimetric detection and stability studies in blood samples," Journal of Chromatography B, vol. 852, no. 1-2, pp. 420-429, 2007.

[10] A. Åsberg, C. N. Hansen, and L. Reubsaet, "Determination of ganciclovir in different matrices from solid organ transplanted patients treated with a wide range of concomitant drugs," Journal of Pharmaceutical and Biomedical Analysis, vol. 43, no. 3, pp. 1039-1044, 2007.

[11] T. Yoshida, R. Takahashi, K. Imai, H. Uchida, Y. Arai, and T. OhIshi, "A simple, sensitive determination of ganciclovir in infant plasma by high-performance liquid chromatography with fluorescence detection," Journal of Chromatographic Science, vol. 48, no. 3, pp. 208-211, 2010.

[12] O. Singh, S. Saxena, S. Mishra, A. Khuroo, and T. Monif, "Determination of valganciclovir and ganciclovir in human plasma by liquid chromatography tandem mass spectrometric detection," Clinical Biochemistry, vol. 44, no. 10-11, pp. 907-915, 2011.

[13] P. J. Ramesh, K. Basavaiah, K. B. Vinay, and C. M. Xavier, "Development and validation of RP-HPLC method for the determination of ganciclovir in bulk drug and in formulations," ISRN Chromatography, vol. 2012, Article ID 894965, 8 pages, 2012.

[14] M. Merodio, M. A. Campanero, T. Mirshahi, M. Mirshahi, and J. M. Irache, "Development of a sensitive method for the determination of ganciclovir by reversed-phase high-performance liquid chromatography," Journal of Chromatography A, vol. 870, no. 1-2, pp. 159-167, 2000.

[15] International Conference on Harmonisation ICH, of Validation of Analytical Procedures: Text and Methodology, 2005. 

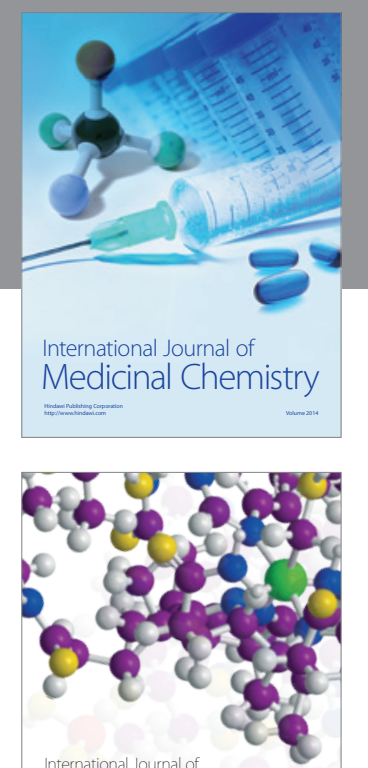

\section{Carbohydrate} Chemistry

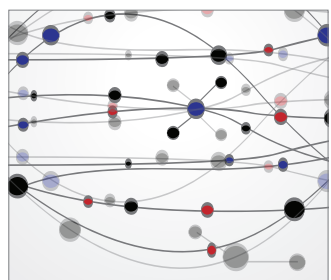

The Scientific World Journal
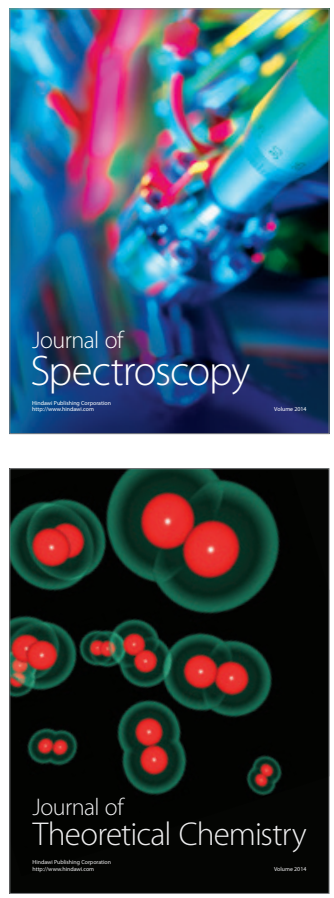
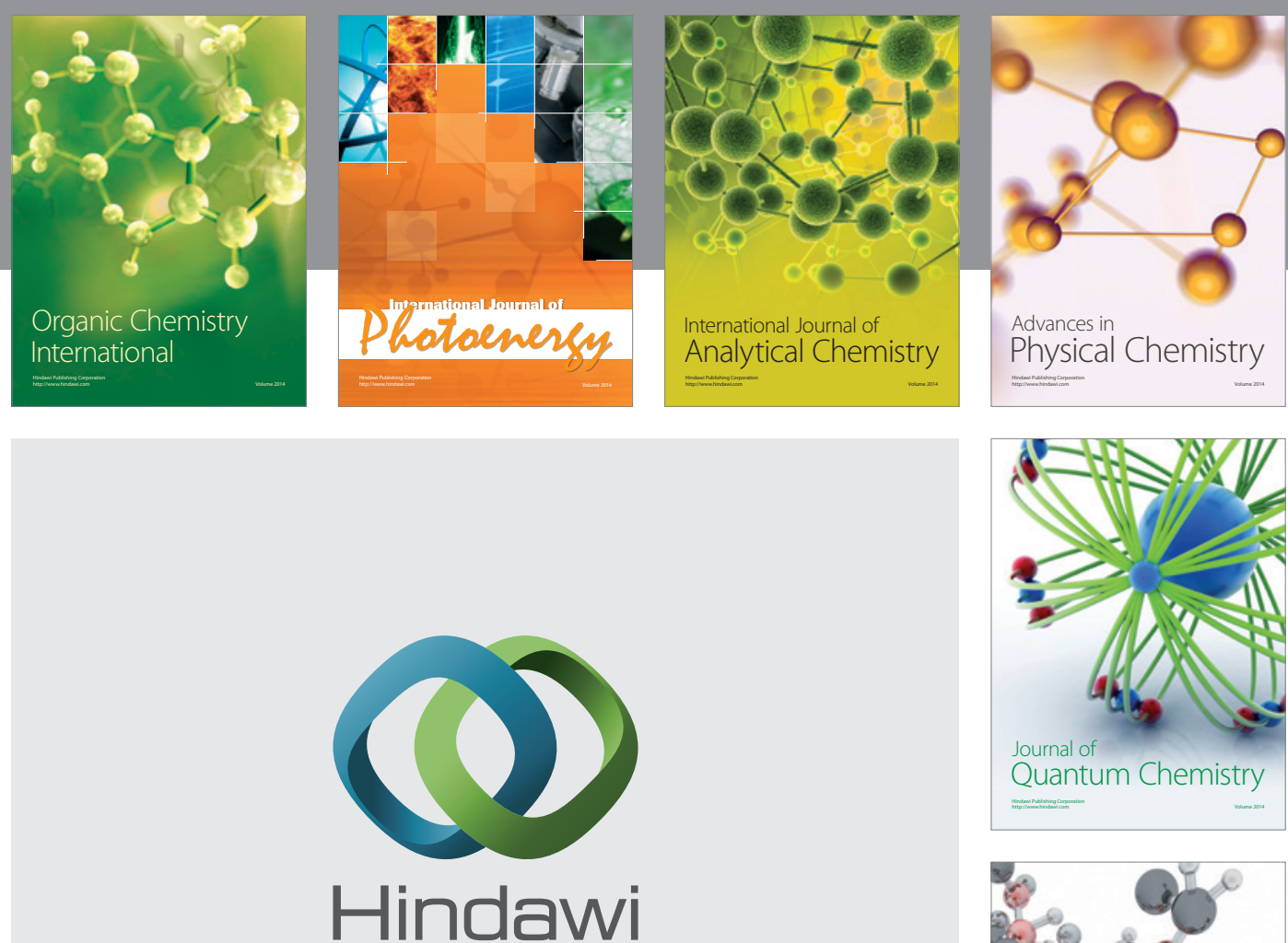

Submit your manuscripts at

http://www.hindawi.com

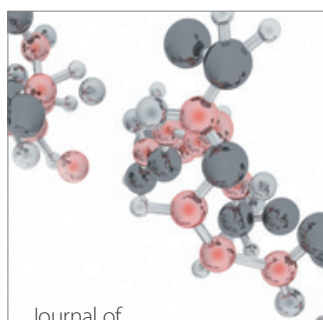

Analytical Methods

in Chemistry

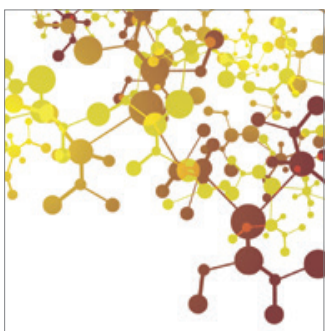

Journal of

Applied Chemistry

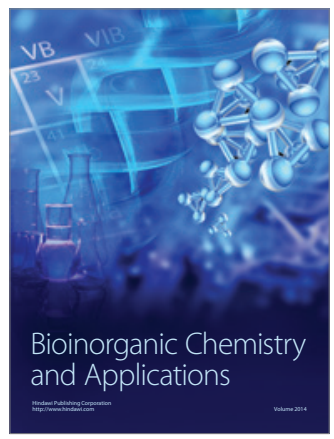

Inorganic Chemistry
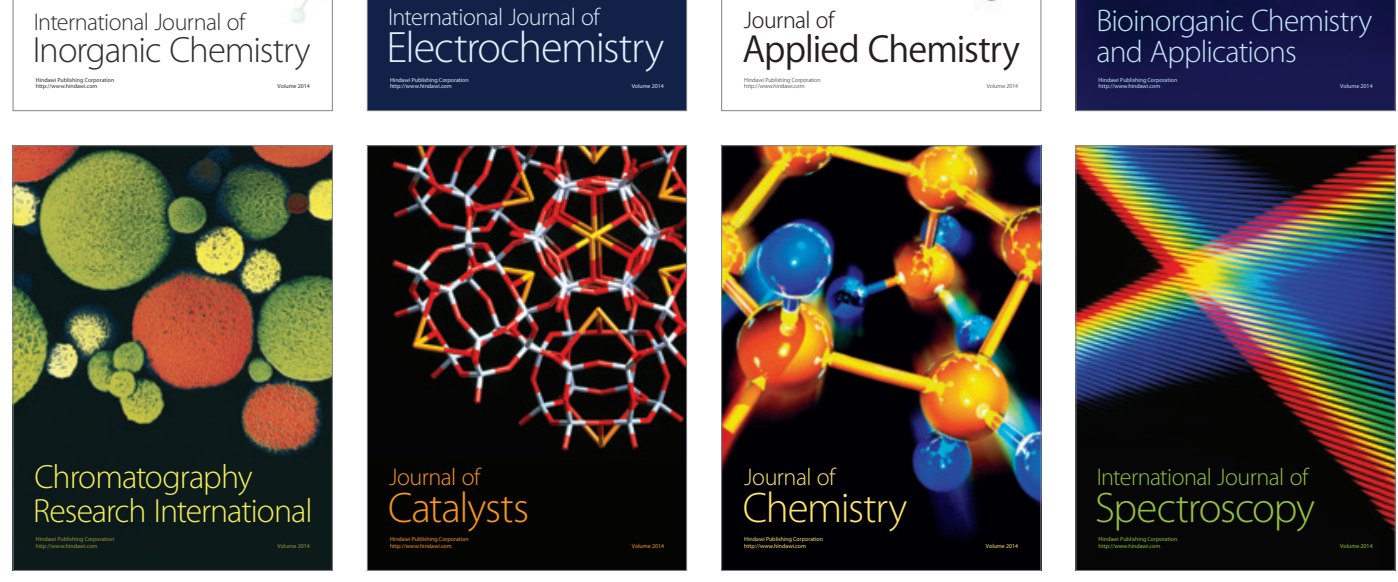